\title{
Research on the Seismic Simulated Vibration Test Machine for the Aseismatic Performance of Elevator
}

\author{
Xiao Lva, Dingdong Zou ${ }^{\mathrm{b}}$, Lei Zhang, Ligui Kang and Jingjie Yuan \\ Chongqing Special Equipment Inspection and Research Institute, Chongqing 401121, China; \\ alvxiao87@126.com, bcq_zdd@163.com
}

Keywords: elevator, aseismatic performance, damage, simulation, inspection.

\begin{abstract}
In view of the running safety in elevator under earthquake, several damage forms of elevator are presented and its analysis model is set up. Then some earthquake protective measures for elevators are proposed. In order to inspect the seismic behavior of the elevator, the seismic simulated vibration test machine of elevator is further analyzed based on the advanced control theory in this paper. Finally, the seismic simulated vibration test machine of elevator is developed and the results show that the method can be applied to elevators. These studies laid the foundation for further exploring the elevator safety performance in earthquake environment.
\end{abstract}

\section{Introduction}

The massive WenChuan earthquake that occured in SiChuan province, China on 12th, May 2012. Measured as magnitude 8.0. It brought enormous loss to people's life and property. According to the information that provided to Special equipment safety supervision bureau of General Administration of Quality Supervision, Inspection and Quarantine in the earthquake, there were 20041 elevators damaged in vary degrees which located in ChengDu, DeYang, MianYang, GuangYuan, Ya'An, ABa. For example, there were 1159 elevators that put into service in MianYang, after the earthquake, 283 elevators went out of service for sever damage upon inspection, 228 elevators without inspection condition due to the collapse of a building or a dangerous house, 511 elevators, $44 \%$ in total. The rest of 330 elevators were judged to be use as guardianship, 28\% in total. General Administration of Quality Supervision, Inspection and Quarantine has quickly organized the safety elimination, inspection and maintenance of the elevators in the earthquake area.

The Wenchuan earthquake enlightened us that safety performance is always the first requirement of the elevator. Today, there are nearly a million new and old elevators running day and night, people are increasingly dependent on elevators. Except for brand, price, quality and function, people are generally concerned about the safety and reliability of the elevator operation and the sense of security in the emergency. The relevant institute of China has lack of research on the behavior of elevators in the earthquake, there have not any aseismic performance in current elevator standards and regulations so far, for example, GB7588-2003. 《Safety Specification for elevator manufacture and installation》 with elevators running in regular condition and effect of safety device, there is no working situation of elevators under earthquake. As the inaccurate prediction and control of the natural disaster of an earthquake, the aseismic performance and requirement of elevators have attracted more and more attention. For the reason, the most easily damaged component of the elevator in the condition of earthquake is introduced and the related failure mechanism is analyzed in this paper. A new elevator vibration simulation test bench based on error feed-forward compensation is proposed. The structure block diagram and the realization method are introduced. In order to verify the feasibility of the method, the related seismic simulation waveforms are obtained by making the prototype.

\section{Earthquake Damage Mechanism in Elevator}

According to the statistics in Wenchuan earthquake, the largest number form of damage in earthquake is counter-weight bracket as shown in Fig.1. Besides, the most dangerous form of damage 
is the crash of counter-weight caused a collision with the lift car. In general, from the perspective of the form of the damage, there are several reasons which caused the elevator damage in earthquake as follows.

a) Under the action of earthquake force, failure or deformation of material has been happened.

b) Strut members of rail, controller and driving machine have been broken.

c) Guidance system has been out of order.

d) Wire ropes in traction machine have been get out of groove.

e) The hook in shaft has been destroyed.

f) Elevator operation in earthquake was not stable.

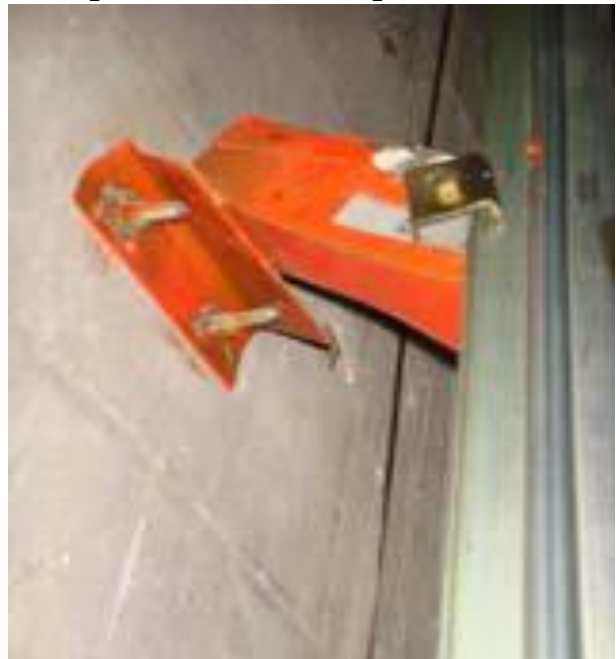

(a) Damage of counter-weight bracket

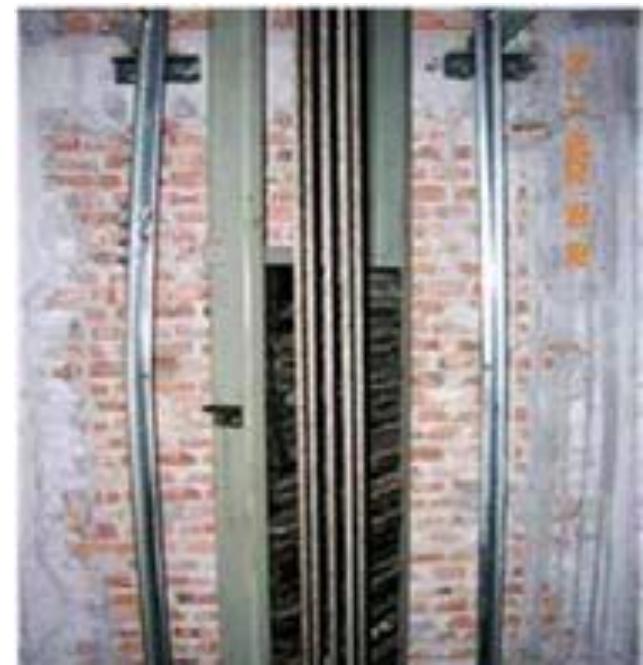

(b) Transformation of counter-weight guide rail Fig.1 Typical Damage in Earthquake of Elevator

Balance weight, guide rail, support frame and guide shoe can be described as a balance weight system. The energy which the building transfered to system through the earthquake wave can be divided into two parts. One part is the deformation energy and kinetic energy of the balance weight system. The other part is damping dissipation of the balance weight system. Therefore, the damage of the balance weight system can be directly caused by the deformation energy.

\section{Structure of Seismic Simulated Vibration Test Machine}

In order to research the seismic behavior of the elevator, there is necessary to inspect the seismic performance test of related parts. Naturally, the seismic simulated vibration test machine of elevator should be developed for further. Due to the seismic simulated vibration test machine is a multi-degree of freedom multi-channel environment simulation system as shown in the Fig.2. The system characteristic varies with the test environment and the state of the DUT, therefore, a certain nonlinear characteristic is presented which would cause error of the output waveform. To overcome the problem, according to the current system transfer function and the desired signals calculated theoretical drive, the error feed-forward compensation method is proposed to get the desired signal.

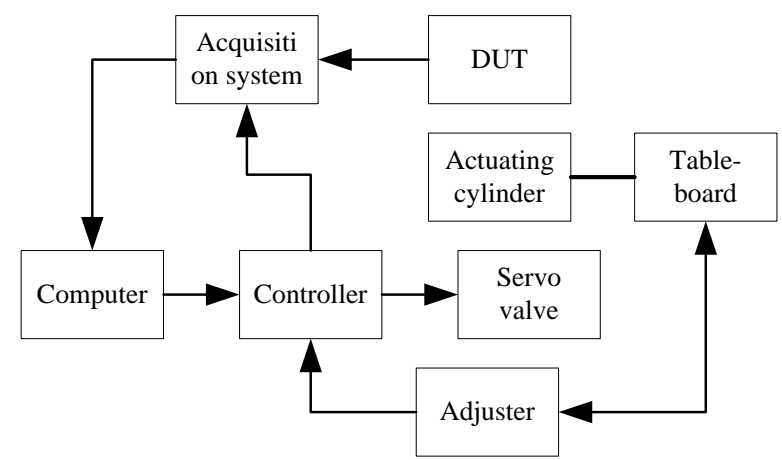

Fig.2 Structure of Seismic Simulated Vibration Test Machine 
In the control system of the seismic simulated vibration test machine, compensation test method is used to make the movement of the vibration table represent the requirements of the seismic wave compensation experiment. First of all, based on the features of current vibration table system, the drive signal of vibration table could be calculated to compensate for the transmission characteristics of the system. Then, according to the error signal, the drive signal could be iterated for reducing the error. The target signal of system could be disposed by D/A, anti-aliasing filter (A/AF) and A/D, then desired signal would be formed which is used as the comparison signal of system response signal. According to the current system transfer function and the desired signal, the driving signal is calculated. The driving signal actuated the mesa after smoothing (SF). After getting the response signal of the system, the error signal is got compared with the expected signal. If the average value of error exceeds demand, drive signal would be corrected by error signal. Then the revised driving signal actuated system again until the error between response signal and desired signal of system meets the requirements.

\section{Experimental Result}

In order to verify the proposed method and study the characteristic of elevator in earthquake, the model machine of seismic simulated vibration test has been developed. As shown in Fig.3, the experimental results of earthquake simulation waveforms are obtained. Fig.3 shows that the simulation waveform is nearly close to the real seismic waveform. The general lumped parameter model introduced in this paper offers more accurate results than the traditional method for dynamic stiffness matrix of foundation is frequency-dependent. It is expected that the general lumped-parameter model will improve the dynamic foundation design. Utilizing this platform, an earthquake simulation platform for elevator with 8 inputs and 6 outputs was constituted. It heightened life span of the screws to several times in vertical and horizontal directions, thus a feasible channel was provided for the earthquake simulation platform to make use of an actuation of electro-mechanical system.

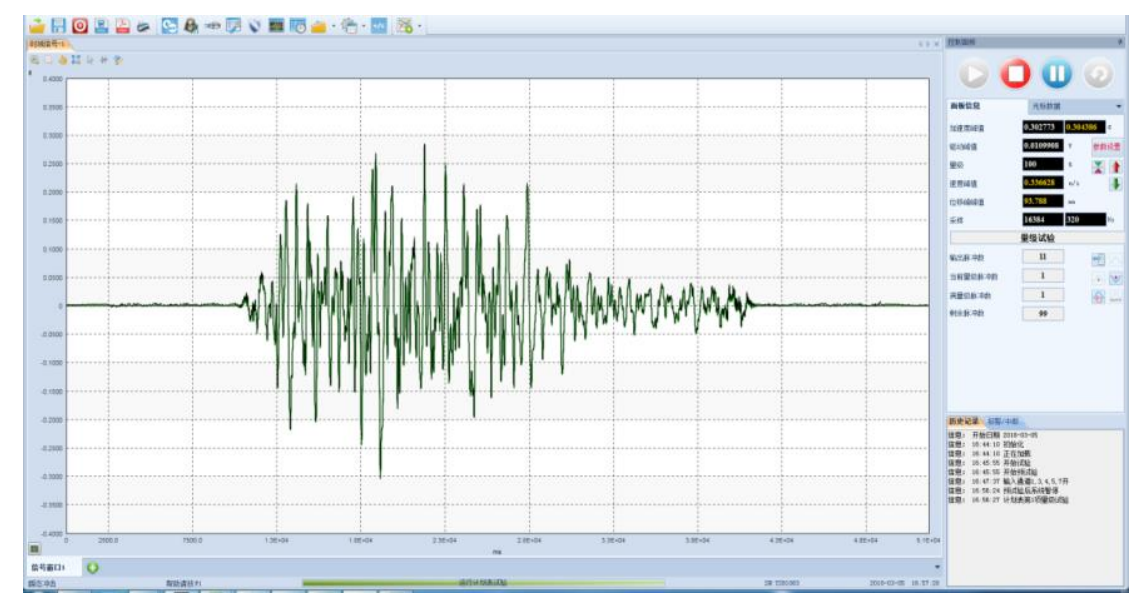

Fig.3 Earthquake Waveforms of Seismic Simulated Vibration Test Machine

A high-speed precision servo system for the machining of a kind of special parts is developed. Based on the analysis of the system, PID regulator is designed and an input revising feed forward error compensation algorithm is proposed. Theoretical analysis and inspection result shows that this input-revising algorithm can improve the accuracy and the transient response of the system.

\section{Summary}

In view of the running safety in elevator under earthquake, several damage forms of elevator are presented and its analysis model is set up. Then some earthquake protective measures for elevators are proposed. In order to inspect the seismic behavior of the elevator, the seismic simulated vibration test machine of elevator is further analyzed based on the advanced control theory in this paper. Finally, the 
seismic simulated vibration test machine of elevator is developed and the results show that the method can be applied to elevators. These studies laid the foundation for further exploring the elevator safety performance in earthquake environment. Then, the research based on the inspection method for seismic behavior of elevator will be studied further.

\section{Acknowledgements}

This work is supported by science and technology planning project of General Administration of Quality Supervision, Inspection and Quarantine of the People's Republic of China (No.2015QK183 \&2015QK189).

\section{References}

[1]. Mikko Siss ala, Taplo Helmola, Matti Otala. Optimization of Lift Car Vibrational Behaviour by Model Analysis[J]. Elevator World, 1985, 901-906.

[2]. K.Nal , W.Fors ythe, R.M.Goodall, Improving Ride Quality in High Speed Elevators[J]. Elevator, 1997,1211-1217.

[3]. Richard A L. Environmental simulation with digitally controlled servo-hydraulic[C]. Proceedings of institute of environmental sciences, 1994,65-70.

[4]. Xu Jinhai, Zhang Shikuo, Zhou Tong. Design and analysis of energy saving and environmental protection type mixing station. Construction Mechanization, 6(2014)97-98.

[5]. WANG Meng-fu, ZHOU Xi-yuan. Modified Pushover Analysis and Seismic Performance Evaluation for Tall Building[J]. China Civil Engineering Journal,2003,36(11):44-49.

[6]. WANG Quan-feng, HUANG Qing-feng, WANG Ling-yun. Theory and Key Technique for the Seismic Design of High-rise Building with Fault Shear Wall[J]. Journal of Huaqiao University: Natural Science,2006,27(2):113-118.

[7]. LOU Meng-lin, YONG Guo-zhu, LI Jian-yuan. Influence of Damping Characteristics on Seismic Responses of Composite Structures[J]. Journal of Architecture and Civil Engineering,2007,24(2):24-29. 